\title{
Ken Jacobs and the Perverted Archival Image
}

\author{
Pablo Gonçalo \\ University of Brasília (Brazil) \\ E-mail: pablogoncalo@gmail.com
}

\begin{abstract}
This paper analyses two recent works by American filmmaker Ken Jacobs that deal with aspects of remediation. The first is A Tom Tom Chaser, in which Jacobs records the telecine process that transforms the classic silent film Tom, Tom, the Piper's Son from chemical into electronic media. The film is riddled with poetic turns inviting the audience to rediscover the medial noise hidden by images. Moreover, Jacobs focuses on the moment of transition from a material medium (the film strip) to the immaterial (the image, the video), so that the noise brings the viewer closer to a perception or brief capture of the medium in itself. Images are both figured and disfigured along this process. The second work is The Guests, an unconventional 3D film in which Jacobs transforms a short take from a Lumière Brothers film by discovering unseen views of the original footage. In his remediation of the 3D technology, Jacobs employs the Pulfrich effect, which allows him to blur the images of the archival film and to create instances of uncertainty between the views coming from the two human eyes. As a result of this procedure, the characters in the film seem to look directly at the audience. The analysis of both films highlights the poetry of the typical manoeuvre by which Jacobs perverts the archival medium, whereupon the viewing mode between media denaturalizes the usual media gaze (framed and representational), focusing on the moment of viewing in itself. This, as a result, favours the medium for what it is and subverts the gaze that expects something representational, discursive, perhaps story-driven.
\end{abstract}

Keywords: archival footage, experimental cinema, Ken Jacobs, remediation, intermediality.

There are artists who can shift back and forth between the two aspects of archival media: between the body itself and its image, between the moment of seeing and the point of view it creates. Ken Jacobs is one of the most interesting cases in terms of such transgressions in perspective. In his book, Breakdowns, Art Spiegelman depicts Jacobs facing the observer. There is a rectangle cutout placed against his left eye, suggesting he has a fragmented view of the observer - who also sees him. From the other side of the gaze, his perspective might be interrupted and cadenced by the frame of his choice. With this play between pictures and 
subjectivities, Jacobs and Spiegelman offer up a sort of gaze that is anything but casual. As he puts the autonomous gaze of the depicted character in evidence, Ken Jacobs not only provocatively inverts (and subverts) the perspectives between observer and observed, but also hints at ways of denaturalizing the gaze by means of sensations and perspectives emanating from the characters, the materialities and immaterialities (the immaterial consisting of a dissolution of would-be representational imagery). [Fig. 1.]

In a verbal portrayal of his friend (and "vanguard filmmaker"), Spiegelman recounts how Jacobs triggered his understanding of paintings as "giant comics panels" whereupon a larger frame may hint at new, smaller-scale images that often escape us. Perhaps that is why Jacobs's picture is fragmented and, interestingly, his right eye is free of the rectangular frame (or canvas), unlike his left eye. However subliminally, what this simple picture of Jacobs proposes is a relationship with the image that both denies and renews one's gaze and, not by chance, introduces an interval and an ontological differentiation between the experience of the left eye and that of the right eye. It generates mediated images that are cutouts rather than a natural instant or perception, something man-made rather than natural. If an image can be fabricated by a point of view, then it can also be dismantled.

In the light of the wealth of Ken Jacobs's experiments with archival images, this paper aims to propose the paths of perversion of the "archival effect" as a mode of perception of the alchemy and the unravelling of ontology of the image woven by the North-American director. I will claim that Jacobs goes beyond just inaugurating other new devices and cinematographic experiences. He also dislodges the experience of image off to a different locus, to another percept. He searches for an experience that lies not in the image, but in the eye; thus, it happens in between the lapses and peculiar intervals created by his perversion of the archival image. Why does Jacobs frame what he sees with his left eye in his portrait? Which image does he want to block? What is the new optical phenomenon that he wants to intimate? If the image no longer resides in the archival film or another support and not even in proper eyesight, where is it in fact realized? Jacobs seems to point out the spectral materiality of the gaze itself and its imaging process, shifting the focus away from a finished image that represents the world as expected. Even more: amidst the perverting acts performed by Jacobs, what is the relationship between writing, inscription, montage, image and materiality?

What is peculiar about the gaze on Spiegelman's frame is that Ken Jacobs's starting point is a pre-captured image, a given image, an archival image wrapped in celluloid film (materiality) as embodied forms of perception. It is an archival 
image that he must open, manipulate, and pervert. Thus, through his optical interaction with the archives, Jacobs proposes something that goes beyond resignification, which has already taken place in the use of the found footage films by means of the so-called archival effect (Baron, 2014). ${ }^{1}$ He seeks hidden images and visualizations from among and inside archival media or virtual crystals, which flourish unexpectedly along the line between material and immaterial, the gaze and infinite imagination (Marks 2010, 10). The perversion of an archival image does not initially occur only between media, forms, and remediations, but it also contaminates gazes, eyes, and materials that were previously regarded as self-evident and uncontested. Additionally, according to Jacobs, the archival image does more than presenting the past: it also develops a future or a space for invention isolated in the gap between the actual medium and its reception by the brain, that is to say, Jacobs gives back to the viewer an image in media res, in the process of becoming something that the brain would read as representational or anecdotic. It is therefore necessary to do a brief review of Jacobs's work up until his recent experiments with 3D technology as seen in his film entitled The Guests (Ken Jacobs, 2014), which will be analysed in the final portion of this article.

\section{On Images beyond Memories, Histories and Archives}

In 1969, Jacobs screened Tom, Tom, the Piper's Son (Ken Jacobs, 1969), a 1905 film produced by both Mutoscope and Biograph that he had found and updated. In an inspired aesthetic turn, he added several new frames as well as decoupage to the old film, changing both its original aspects (such as meaning, narrative and message) and aesthetic principles. ${ }^{2}$ This famous found footage film influenced some remarkable experimental and contemporary filmmakers such as Michael Snow and Peter Kubelka, among many others. In addition, Jacobs also approached

1 Here we refer most of all to the good work of Baron (2014), who understands the archival and the entire found footage experience as a form of aesthetic imagination of history. We will argue that Jacobs's use of the archival material is closer to an ontological debate not just about the image but also about sight itself. In my view, the perversion of the archival image happens precisely in that moment of transition: when an archival image ceases to be strictly historical in order to become an optical, historical and perceptual aberration.

2 Since 1961, however, Ken Jacobs has been directly involved with the Anthology Film Archives group, which gravitates around Jonas Mekas and his circle of friends. He has also been conducting research, screenings, debates, and experimental film productions. The over 30 films that Jacobs has directed can be divided in two different groups. First, there are his experimentations with archival images, in which he focused on the "cinema of attractions" period of early cinema. The second group comprises his work with 3D and the so-called Nervous System, which is detailed on the third part of this article. 
early cinema by going beyond the established interpretation that restricted this period as pre-narrative (and pre-historical) cinematography. By emphasizing the visual attractions of films like Tom, Tom, the Piper's Son (Ken Jacobs, 1969), Jacobs directly influenced the academic research of Tom Gunning and Charles Musser, whose works modernized the interpretation of early cinema.

It is worth noting in the found footage of Tom, Tom, the Piper's Son, how Ken Jacobs made small yet notable alterations in the views, the cuts and the highlights of the myriad images and small frames that make up the long shot of the 1905 film. He not only edits the found archival film, but also creates interest by reshooting it to capture his projections on screen, thus establishing a new meeting point between the projector and the camera. At the interregnum of this meeting - the intervals that are so deftly emphasized by the editing, oscillating between stills and the movement in the archival images (Røssaak, 2011) - Jacobs expressively handles the archive. Consequently, he yields a presence, not that of the "archival effect," but of the materiality (and visuals) of the archival. By putting an emphasis on the tangible quality of the archival image, and by performing the process or mechanism of imaging, the filmic effect folds in on itself neither as image nor as experience, but as materiality.

Therefore, from early on, Jacobs's intentions were to awaken real aberrations within archival images. He duplicates the archival film, which gets deliberately reproduced as a dangerous simulacrum. The images we see in Tom, Tom, the Piper's Son are no longer copies nor reproductions of the recorded medium and the archive of that recording. They are images that call back to a possible remembrance, something that has dissipated in the process of being disfigured by the adulterated editing, which takes them beyond the archive and intimates historical and visual transgressions. Those hidden and volatile ghosts are aberrations that nevertheless create a new mechanism for the archival image, which, when adulterated, makes up new images in between the shooting and the projection, leading to new optical discoveries. The poetics and performance of the archival film is a constant and perpetually renewed gesture seen throughout Jacobs's work. It can be seen on the threshold of languages and media, along the border of the gaze that moves between visible forms and mediations (internal and external) of optical media. This is why Jacobs divides the film into three parts: the full section; then the misconfiguration of this, cutting, and fragmentation; then the perversion and final presentation of the same section, now with the an essentially changed image form and visual experience. Jacobs's poetics of archive subversion is an optical-material turn. 
The second approach consists of Jacobs's experiments known as The Nervous System films - again, directly related to archival images. However, Jacobs did something more radical when he inverted the sense and directions of the archival frames, thus confronting the audience's habits of image formation, which might occur in the gaps between eyes and brain. Ken Jacobs explained the main goal of those experiments in his Notes on the Nervous System: "[t]he Nervous system consists, very basically, of two identical prints on two projections capable of singleframes advance and 'freeze' [...]. The twin prints plod through the projections, frame... by frame..., in various degrees of synchronization. Difference makes for movement and uncanny three-dimensional space illusions via a shuttling mask or spinning propeller up front, between the projectors, alternating the cast images." (Pierson 2011, 16.) [Fig. 2.]

Jacobs's 2005 film, Ontic Antics Starring Laurel and Hardy: Bye, Molly, is possibly his most famous and descriptive Nervous System experiment. In one of the first frames of the film, Jacobs shows the wheels of a train spinning in different rhythms and directions. Sometimes the image bifurcates then fuses two frames into one. Sometimes the image is paused and the audience hears an astonishing soundscape, which is also inconstant. Although Ontic Antics is not a 3D film, it was directly influenced by the $3 \mathrm{D}$ principle while in the making. This means that Jacobs emphasized the intervals between frames, in the cinematographic image, as well as the lapses between eye perception and the brain registering an image. In Ontic Antics Jacobs edited the archive of his found footage film according to the rhythm of an old locomotive and, inspired by this cinematographic imagery; he forced the audience to see something that had not previously been present (neither perceived nor imagined) in the frames of the archival.

In both his experiments with archival films and the central nervous system, Jacobs establishes a double and paradoxical process that shifts assertively between archival film and the images they convey. On the one hand, he struggles in poetic agony with the index, as if denying the fact that the index can suggest a single image of an event. By cutting, tiling, and even hacking the index in the archival films, he rejects the unity of the information and images therein disseminated; ${ }^{3}$ he

$3 \quad$ My proximity to Jacobs's archival image perversion and its hacker turn unfolds in a sense upon how the distortion of a certain "original" ontology ends up generating new possible and potential worlds. In that sense, it is worth sharing the second paragraph of the hacker manifesto, which aims to offer an ample perception of that turn: "Whatever code we hack, be it programming language, poetic language, math or music, curves or colourings, we create the possibility of new things entering the world. Not always great things, or even good things, but new things. In art, in science, in philosophy and culture, in any production of knowledge where data can be gathered, where information can be extracted from it, and where in that information 
ends up mistrusting the image he wants to convey with such confidence. In Ontic Antics, this phenomenon may be noticed as we see two duplicated and divided images, one on each side of the screen, triggering a fusion and getting mixed with the images in the center, resulting in a third view. Shattered, broken, the image there lies not just in the archival footage, and not at all restricted to the right or left eye, as it cannot even establish unity. The image falls apart entirely. It is recreated in the body, inside the brain; it "happens" - fast and volatile, within the same temporal lapse that gets "archived" within the body of the spectator. The image, in this way, is "archived" inside the body as a result of a direct contact of the filmic "materiality" with the spectator's corporeality. This moment leads to more doubts than certainties. Would it not be a sort of perversion of the image?

On the other hand, along with this mistrust, Jacobs begins seeking hidden images - virtual but present - condensed among poorly developed nitrate salts; images that were sometimes randomly captured, yet are still able to resurrect from the shadows of the archives. This pursuit is permeated by a faith in finding images, however evasive, temporary, distorted or hallucinated. This is at the line that separates the index from the event when Jacobs excavates, adulterates and deprogrammes images. It happens in the intermediate places of perception, where Jacobs traverses the crystals of time and brings the material up-to-date, as if poetically touching the fractals of the archives, or the virtuosities of the image. If, as Laura Marks reminds us, the image establishes a relationship between information and infinity, then Jacobs annuls the feelings and the senses of perception and invites the spectator to experience new bendings of space - on the other side of the archival, and in the perception of images, unusual temporal twists pointing to the future that take place in the brain of the spectator. As a result of this manoeuvre, the perception of the archival image as memory or as any metaphysical redemption of the past is not entirely possible. The archival film and its ontological information from a remote past are torn apart, de-programmed and, as the index is not persistent, one may go on to view something beyond the index or its materiality, something that can only be concocted in the brain in reaction to the subversion of imaging expectations. The peculiarity lies in the fact that those volatile, aberrant images are nevertheless material.

new possibilities for the world are produced, there are hackers hacking the new out of the old. While hackers create these new worlds, we do not possess them. That which we create is mortgaged to others, and to the interests of others, to states and corporations who control the means for making worlds we alone discover. We do not own what we produce - it owns us." (Wark 2004, 002.) 
Jacobs provokingly throws us into another environment in order to help us go beyond an understanding of the archive restricted to testimony, as addressed by, for example, Agamben (1998) or Sarlo (2007). The audiovisual shimmer in this new cut points to what is to come, and in the wake of Derrida's archival fever, the archival generates its own deviations, speculums, and speculative aberrations about what is beyond the index and the aura of the occurrence. For Jacobs, the archival is above all multiple, multifaceted, and malleable - an optical phenomenon that takes us to other spatial-temporal spirals.

Like a hacker immersed in analogue media, Jacobs disrespects the archons of audiovisual archival films. Archons are understood, according to Derrida, as the power of constriction that makes archives possible - the preconceived, patriarchal, and authoritarian ideas that engender secondary, derived, and preformatted concepts and perceptions. To Jacobs, this power is above all optical: technological standards that invent (and restrict) the technical possibilities found in the act of perception and observation. Therefore, Ken Jacobs perverts the optical archons and their data - the moulds of perception - along with the technical-sensorial configurations that make the audiovisual gaze possible as well as historically or technologically restricted. Yet, what does it mean to say that Jacobs perverts archival images?

To be more specific, perversion reappears as a poetic mode in Jacobs's more recent films such as The Guests. In any case, what we suggest as a "poetics of perversion" consists of a subversion of an original grammar and meaning, which, concerning the archival images and their "effect" (cf. Baron 2014), approaches a redemption of a past long gone and therefore metaphysical. On the one hand, Jacobs challenges the spectators to see, in the act of re-watching an archival film, an inapprehensible past in relation to the past itself that the archival film attempted to record. On the other hand, the archival image itself is viewed directly in its materiality. There is no more past, or an act of temporal differentiation from a present gaze that updates the archival, but an ebullience of the crystals of the past that generate images of an offbeat future. This is the essence of the perversion of the archival. By being perverted, the archival itself bifurcates and makes possible mediations of perspective between the distinct natures of the image, between the gaze of the observer and the singular feeling by the observer of also being gazed upon (Castro, 2002). 


\section{A Tom Tom Chaser and Some Archival Boundaries}

Jacobs qualified A Tom Tom Chaser (Ken Jacobs, 2002) as a scanning improvisation. In an extremely simple manner, the film uses the archive of Tom, Tom, the Piper's Son and follows its telecine process of transferring the "original" chemical material to an electronic image. Using a similar aesthetic principle, Jacobs also reframes the original takes of the Mutoscope and Biography film archive. However, in A Tom Tom Chaser the image blur is much stronger and takes center stage. [Fig. 3.]

By emphasizing its process of material and mediatic transformation, Jacobs refrained from assigning a locus for the archival. Thus, he uses the transformation of the archival image to invite the audience to invent their gaze. The material is neither an original archive nor a future archive in the process of becoming. It is only a simple and displaced archival image. Peculiarly enough, the gaze of the audience has no other possibility: it must occur in between medias, within a certain boundary, and without specific media (or form) configurations. [Fig. 4.]

Wittily edited, A Tom Tom Chaser creates fast aesthetic perceptions that sometimes impose a specific medium upon the audience; thereafter it casts the same image into another media materiality and then alternates the perception of the image between different frames. This reframing occurs in between media, consequently blurring the image. On top of that, the eye rarely has enough time to reframe between these media. This means that the eyes need more time to capture, focus, and see (or believe they are seeing) an image beyond media and materiality.

Directly or indirectly, the dynamics created by Jacobs interact with the remediation concept elaborated by Jay David Bolter and Richard Grusin, who state that remediation is a transference and refashioning of the content from one medium (and the perception of the medium) to the configuration of another medium. However, remediation might happen in two different instances and in aesthetic or technological turns. The first is known as immediacy, which creates a first person point of view and a virtual reality effect. In visual media, immediacy puts viewers through an immersive experience in which seeing simulates a sense of reality. In their genealogy of immediacy, Bolter and Grusin refer back to the Renaissance perspective when viewers saw through the image and had a feeling of believing what they saw. Thus, immediacy constructs a natural sensation and an "unmediated presentation."

On the other hand, hypermediacy is a construct that presents viewers with the perception of the media configuration itself. It means that the viewer can 
simultaneously observe the representation as well as the media used to enact this representation. Thus, hypermediacy flirts constantly with fragmentation, heterogeneous media, interruptions, and indeterminacy. "In all its various forms, the logic of hypermediacy expresses the tension between regarding a visual space as mediated and as 'real' space that lies beyond mediation" (Bolter and Grusin 1999, 41).

Jacobs's remediations in A Tom Tom Chaser (as well as in most of his works) have a more precise dialogue with the hypermediacy tradition. However, for very brief moments, Jacobs invites viewers to believe in what is known as the archival effect, which is a sense of past and reality while casting the gaze upon the frame. The Tom Tom archival image oscillates between chemical and electronic perception, and Jacobs inserts both remediation and hypermediacy between media. In a provocative manner, Jacobs asks the audience to at once believe and doubt what they are seeing.

Where the scanning process erases traces of remediation in a digital age, Ken Jacobs does the exact opposite. In between archival images (and hypermediacy), A Tom Tom Chaser offers precisely a scanning-image. When an image is liberated from its material support, it occurs only inside the brain in a delay, as an illusion. However, and paradoxically, it is precisely when the image, during the interval of its remediation, becomes ethereal that it reaches the viewer, gets embodied and becomes an archival image. Jacobs offers both a meeting and a mismatching of the material and the viewer's body; and the material consists of matter in its fault, its ephemeral and fully realized blank, matter before matter, matter before memory. Without specific materiality, the image is restricted to a phantom, or a simulacrum that almost becomes an autonomous figure. Again, that is why it is a perverting act, since the image for Jacobs is always essential and extremely material yet never ceasing to be imaginative in an offbeat, unpredictable and unsettling way.

\section{The Guests: Perspectivism, Paces and Paths of Figures}

Finally, what would this material and poetic perversion insistently woven by Jacobs be? By exploring the transition and the buffer zones between different media and materialities, Jacobs ends up intertwining images of different nature, which approaches something that can be described with the concept of multinaturalism, used by Viveiros de Castro $(2002,377)$, in the field of anthropology. (This refers to the belief that lies at the core of the mythologies and cosmologies of indigenous 
American cultures, namely that that animals are former humans. We are, of course, only borrowing this anthropological concept; nevertheless, it allows us to enlighten some of the phenomena of perspectivism. Jacobs is frequently referred to as an alchemist; and we hereby stress his shamanic side in order to outline distinct imagistic perspectives that are completely isolated from one another.

And that is where the discussion encounters the debates about an ontology - anthropological and imagistic - that favours incompleteness, intervals and inconstancies between modes of perception over a properly rounded, defined form with a single framing. The perversion would occur during that passage between opposite perspectives. When animals cease to be human, and when humans, by means of their shamanic rites, abandon their bodies to tour animal cosmologies, a twist of perspective occurs which creates the opportunity to reconsider the world. Perversion, on the other hand, and in Jacobs's poetics, happens during the process of the sensible transfiguration of matters which get dematerialized, which abandon their original bodies in order to, deprived of bodies, like animistic entities, create a visual vertigo that approaches aberration, leading to something inaccessible, almost forbidden. Perversion would be the poetic act of traversing, of passage, by which the viewer would gain access to a sort of impossible nature, an entirely other language, which, much like an Eden, has no more ontological possibility of interacting, imagining or even seeing. And that is the kind of perversion - since perspectivism would be essentially heretical - that we see in most of Jacobs's films and archival experiments.

Recently released in the festival circuit only, The Guests is one of Ken Jacobs's latest films and it is directly screened in 3D technology. This is the first time he uses a 3D camera and 3D glasses in his work. However, as stated earlier, Ken Jacobs has been experimenting with 3D concepts since the 1970s, his Nervous System films can be seen as an intimate continuity between both moments.

As in his other works, Jacobs uses an archival and a found-footage chemicalbased film as a starting point for The Guests. The original Lumière film is only a few minutes long and merely registers the arrival of guests at the wedding reception of the Lumières' sister. It is a little-known film by the Lumière brothers and it has a peculiarity that is widely explored in the framing: guests appear lined up before the Lumières' camera and speed up, almost one by one, in a sequence that allows for the exploration of a peculiar depth-of-field. The first perversion Jacobs does in the Lumière film is the radical extension of cinematic timing using slow motion to extend second-long frames into minutes. By alternating rhythm and movement, Jacobs transformed the few minutes of the Lumières' film into a 
90-minute experience. This radical extension dilates the presence of the figures, of the guests, who walk step-by-step, frame-by-frame, through the Lumières's camera and the 3D glasses. [Fig. 5.]

The second perversion is directly related to the Pulfrich effect, which, as Ken Jacobs once affirmed, is one of the key concepts that influenced his 3D experiments. The Pulfrich effect occurs when the two eyes send image signals to the brain in different time frames. The gap in this case is totally different from retinal persistence due to which the internal movement of the cinematographic apparatus and frame opens and creates an illusionary image. The gap of the Pulfrich effect occurs very subtly in and between the two eyes and the brain. It creates a delay in the picture and small differences in light, thus inserting a pendulum or time lapse between the eyes and the brain, which extends the duration of perception from one image to another. The odd thing is that the Pulfrich effect has often been used to create the three dimensional effect. In The Guests, Ken Jacobs does the opposite: he uses 3D glasses and employs the Pulfrich effect to provoke distrust in the act of seeing; he blurs the view and creates a misty three-dimensional environment. By this, the 3D becomes opaque. Consequently, the imagistic pendulum woven by Ken Jacobs establishes a spatial disparity in moving objects. Therefore, The Guests is a film in which space is not a projection, but an invasion. It is exactly in that aspect of invasion that the multinaturalism of image - between different materials and temporalities - gets transfigured into perspectivism. Instead of just seeing, as the usual projection of Renaissance perspective, the viewer goes on to feel observed, seen, followed by the gaze of those objects, those sculptures marching in a slow and precise movement toward the other side of the frame, of the field, of the shot and the traditional cinematic space.

Ken Jacobs combines the length of an archival footage with the Pulfrich effect and, little by little, adds 3D. Thus, he perverts the so-called archival effect and does something more radical than remediation. Just like in Adieu au Langage (Godard, 2014), Godard's latest film, one can notice in Jacob's work a hypermediacy experiment with 3D. In this, an opaque 3D phenomenon "happens" in between the eyes and the brain and produces a strange feeling when using the 3D apparatus, which is itself perceived and experimented by the audience. However, The Guests gradually becomes a film of effects that hints at counter effects. It is as if it played against its own device: it starts with the 3D, showing unusual or nearly invisible frameworks and frame details. By this, the Lumières's film refers back to the brushstrokes of an impressionist painting (echoing Godard, who once called 
the Lumière brothers the last impressionists). The audience begins to perceive faces, tiny details, delicate gestures and unusual movements or image-times. In this film, Jacobs creates a 3D experience similar to kinetic art and uses a play of colors and depths that reminds us of a Mondrian painting. We are looking at art that highlights surfaces.

It is as if the ghost-like imagery recorded by the Lumière brothers had sprouted volume, shape, and forms that, once alive, presented autonomous effects. This is a sculptural film. Gradually, between one pendulum and another, between the ontological and vertigo images, there is the feeling that the audience is not composed of viewers watching ghosts, but rather the exact opposite. The viewers are being seen and observed from the other side of the crystal-images. This spatial disparity of The Guests makes possible the survival of the images (Nachleben), as Aby Warburg described it (Didi-Huberman 2002). However, Jacobs still debates a cinema that abolishes the dynamics of Renaissance perspective and reveals a form of sensitive image organization that denies the original projection or point of escape. Ken Jacobs eventually creates fissures in the ontology of media filming within the Lumières's archive, and perverts it. His version is the opposite of archeology; it is nostalgia and it is what has been called the archival effect, because he points to the future, to images that demand to be created. The remediation instant in The Guests can be considered an almost impossible dismediation of the archive. It is a heretical act that goes beyond aesthetics. It is a form of salutary disrespect toward time and the movements inscribed inside the archival material. Finally, when this unprecedented experience ends, we toast to the vitality of our retinas while, distant and guarded, Ken Jacobs greets us with this unique visual heresy.

In bringing this article to a close, I would like to comment on a last picture in which Ken Jacobs inserts his own image into the archival footage, and appears as an audiovisual figure. In As I Was Moving Ahead Occasionally I Saw Brief Glimpses of Beauty (Mekas, 2000), Jonas Mekas shows and comments on an archival recording from the 1970s at his house. There are intimate and festive moments that celebrate the delicate quality of a daily life that is easygoing, pleasurable and grounded. Mekas, surrounded by friends in his apartment, tells them about a time when Jacobs, Bible in hand, read, commented and talked about one of its passages. The scene has no sound and, even as an archive or index of this occurrence, the audience can only speculate as to what Jacobs said. Even so, the scene is truly revealing. Jacobs, Mekas and Spiegelman all have Jewish origins and seem to intentionally play with images, memories and archival film 
in a playful effort to bring back to life something that already happened - from the index to real imagination, something already given, inscribed and archived in this great support that we can, somewhat mystically, call world.

However, Jacobs's Jewish turn leads back to a play of gazes, images and figurations that is deeply rooted in the prohibition of idols in that religion. By referring back to an image that is not there, evident and stark, in the archival film, Jacobs turns to a way of seeing that seeks to transpose the veil of image itself and intimates a non-figurative vision, truly different from the Christian, metaphysical idealization. As Mondazain very deftly stressed, the veil of image is, for the Jewish Torah tradition, an evident materiality, a stance of the literal, "a pure screen of the legible that cannot, in any shape or form, be in the sphere of inscription of the visible" (Mondazain 2003, 42). It is the invisible visible, beyond figurative image that this way of seeing, deeply rooted in Jewish tradition, ends up establishing. In that vein, we can say that at the core of the archival perversion, in Jacobs's works, lies an eagerness of surpassing a particular way of seeing the archival that is essentially figurative and metaphysical. The perverting act flirts with the prohibition of the idol image by Judaism as much as with a turn, a different way of seeing that seeks, aesthetically and poetically, to enliven the invisible that lies beyond the image.

If everything is written, then a certain original aura can be awakened, if only to get in touch with the archives of the world. In this way of thinking, writing is less about inventing something new than about rewriting (or reviewing) what has already been written, stored and inscribed. It is within this fairly religious environment that Ken Jacobs's archival film subversions persist in implementing the act of seeing beyond the image - in transcending its sacredness - and beyond its physical, material and media support. These are profane gestures that embody an ethical presence. If the audiovisual archive creates a world that has already been given to the viewer, then Ken Jacobs seeks to see through it and reveal what escaped and did not get stored. It is as if, from the creation of the world, we pass through cinematographic imaging to the fundamental, silent and unmoving figures inside, and that little by little we are accompanied by their ghosts until, in a psychotic schizophrenia of images, they begin acquiring optical and sculptural autonomy. The summary of Ken Jacobs's poetic perversions unfolds in an audiovisual world created by the very creatures that inhabit the archival media where the figures are no longer passive, but control the previously autonomous gaze of the viewer. They are figure-guides that lead us to a world which, indeed, we can see (and read), but can never inhabit. The paradoxical effect of those 
imagistic experiences lies in their power and reveals itself in the vertiginous instances and hallucinations that come from poetically giving up control of our gaze. At the end of each session, such images indelibly inhabit our memory. This is the heretical pleasure of an optical heresy that the perversion of archival film offers to its spectators.

\section{References}

Agamben, Giorgio, 1998. Homo Sacer: Sovereing Power and Bare Life. Stanford, California: Stanford University Press.

Baron, Jaimie, 2014. The Archive Effect: Found Footage and the Audiovisual Experience of History. New York: Routledge.

Belisle, Brooke, 2014. Depth Readings: Ken Jacobs's Digital, Stereographic Films. Cinema Journal vol. 53, no. 2 (Winter): 1-26.

Bolter, Jay David and Richard Grusin. 1999. Remediation: Understanding New Media. Cambridge, Massachusetts: The MIT Press.

Castro, Eduardo Viveiros de. 2002. A inconstância da alma selvage [The Inconstancy of the Indian Soul]. São Paulo: Cosac \& Naify.

Derrida, Jacques, 1995. Mal d'archive: une impression freudienne [Archive Fever: A Freudian Impression]. Paris: Gailée.

Didi-Hubermann, Georges, 2002. L'image survivante. Histoire de l'art e temps de fantômes selon Aby Warburg [The Surviving Image: History of Art and Phantoms of Time According to Aby Warburg]. Paris: Minuit.

Gunning, Tom, 1989. Films that Tell Time: the Paradoxes of the Cinema of Ken Jacobs. In Film that Tell Time: a Ken Jacobs Retrospective, ed. David Schwartz, 3-11. New York: American Museum of Moving Image.

Marks, Laura. 2010. Enfoldment and Infinity: an Islamic Genealogy of New Media Art. Cambridge, Massachusetts: The MIT Press.

Mondazain, Marie-José. 2003. Le commerce des regards [The Trade of Looks]. Paris: Éditions du Seuil.

Pierson, Michele. 2011. Ken Jacobs - a Half Century of Cinema. In Optic Antics: the Cinema of Ken Jacobs, eds. Michele Pierson, David E. James and Paul Arthur, 3-25. Oxford: Oxford University Press.

Røssask, Eivind. 2011. Acts of Delay: The Play between Stilness and Motion in Tom, Tom, the Piper's son. In Optic Antics: the Cinema of Ken Jacobs, eds. Michele Pierson, David E. James and Paul Arthur, 96-107. Oxford: Oxford University Press. 
Sarlo, Beatriz. 2007. Tempo Passado: cultura da memória e guinada subjetiva [Time Past: Culture of Memory and Subjective Turn]. São Paulo: Companhia das Letras.

Spiegelman, Art. 2008. Breakdowns: Portrait of the Artist as a Young \%@ $\mathcal{F}^{*}$ ! New York: Pantheon.

Wark, McKenzie 2004. A Hacker Manifest. Cambridge, Massachusetts and London: Harvard University Press.

\section{List of Figures}

Figure 1. Ken Jacobs in Breakdowns: Portrait of the Artist as a Young \%@?*! by Art Spiegelman. Figure 2. Ken Jacobs: Optic Antics Starring Laurel and Hardy: Bye, Molly (2005).
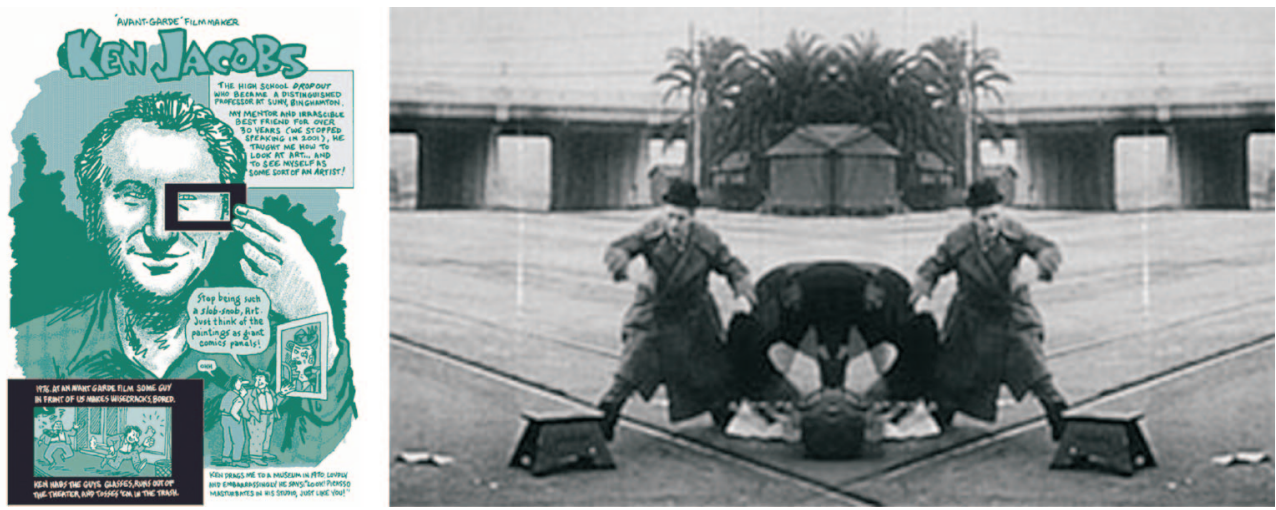

Figures 3-4. Ken Jacobs: A Tom Tom Chaser (2002).
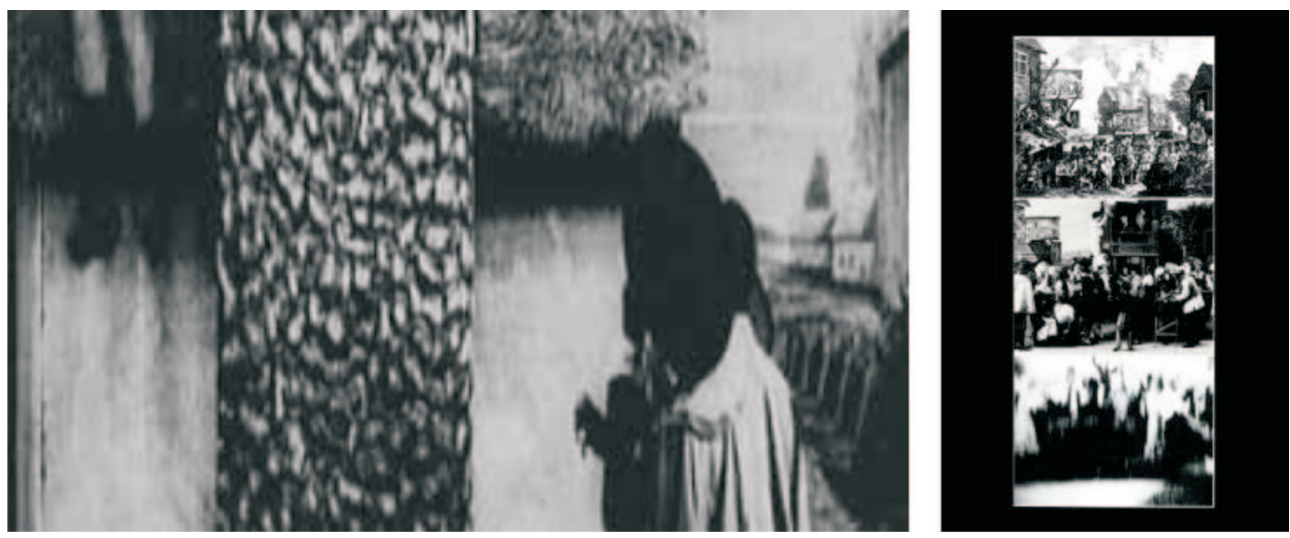
Figure 5. Ken Jacobs: The Guests (2014).

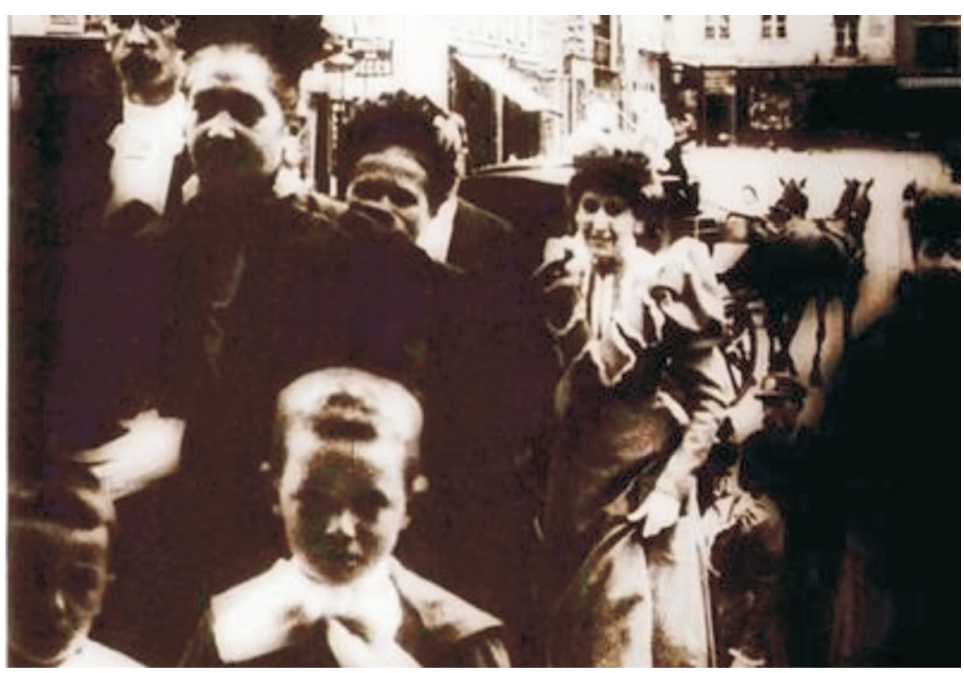

